
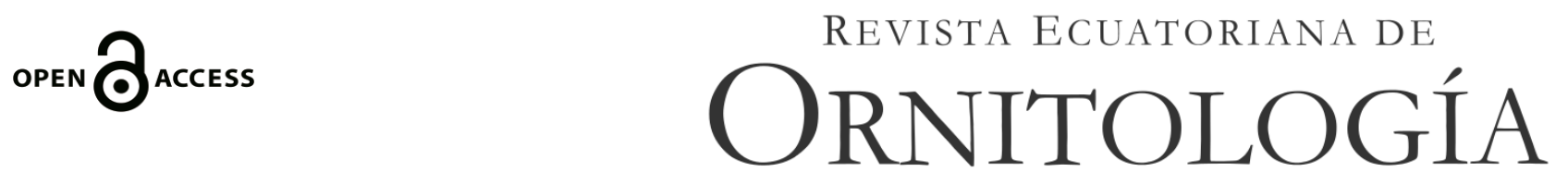

NOTA/NOTE

\title{
Nuevo registro de anidación y descripción del nido del Mosquero Real del Pacífico Onychorhynchus occidentalis (Passeriformes: Tyrannidae) en El Oro, Ecuador
}

\author{
Karla Pezo \\ Universidad del Azuay, Escuela de Biología, Ecología y Gestión, Av. 24 de Mayo 7-77 y Hernán Malo, \\ Cuenca, Ecuador \\ Editado por/Edited by: Diego F. Cisneros-Heredia \\ Recibido/Received: 10 agosto 2017. Aceptado/Accepted: 21 noviembre 2018. \\ Publicado en línea/Published online: 01 mayo 2019

\section{New record of nesting and nest description of Pacific Royal Flycatcher Onychorhynchus occidentalis (Passeriformes: Tyrannidae) in EI Oro, Ecuador}

\begin{abstract}
The Pacific Royal Flycatcher Onychorhynchus occidentalis is distributed in Ecuador in the humid and semi-deciduous forests of the southwestern Andes. These forests are currently under pressure from anthropic activities, mainly the expansion of the agricultural frontier, making it vulnerable to extinction in global and national scales. There is limited information about its behavior, ecology and especially about its breeding biology. In this note I provide information on its breeding behavior, describing parenteral care and describing its nest for the first time in the province of El Oro, southwest Ecuador. This note contributes to knowledge about the species and might be useful for future conservation plans.
\end{abstract}

Keywords: Behavior, nest, breeding biology, Onychorynchus occidentalis, dry forest.

\section{Resumen}

El Mosquero Real del Pacífico Onychorynchus occidentalis se distribuye en Ecuador en los bosques húmedos y semideciduos del suroccidente de los Andes. Estos bosques actualmente se encuentran bajo presión por actividades antrópicas, principalmente debido a la ampliación de la frontera agrícola, lo cual hace que esta especie se considere vulnerable de extinción en escala global y nacional. Existe limitada información sobre su comportamiento, ecología y especialmente sobre su biología reproductiva, por lo que en esta nota se detalla información sobre su comportamiento reproductivo, se describe el cuidado parental y se da una descripción de su nido por primera vez en la provincia de El Oro. Esta nueva información contribuye al conocimiento sobre la especie y puede ser incorporada en futuros planes de conservación.

Palabras clave: Biología reproductiva, comportamiento, nido, Onychorynchus occidentalis, bosque seco.

El género Onychorhynchus (Tyrannidae) agrupa cuatro especies distribuidas en el Neotrópico, desde el sur de México hasta el sureste de Brasil (Sample et al., 2016). Onychorhynchus occidentalis tiene distribución restringida a bosques húmedos y deciduos de tierras bajas en la región Tumbesina de Ecuador y del norte de Perú (Sample et al., 2016). Esta región es importante para la conservación debido a su alto grado de endemismo (Best et al., 1996). En Ecuador, O. occidentalis ha sido registrada desde el norte en la provincia de Esmeraldas hasta el sur en la provincia de El Oro, con un rango de elevación desde el nivel del mar hasta $600 \mathrm{~m}$ (Ridgely \& Greenfield, 2001; McMullan \& Navarrete, 2017); aunque, existen reportes hasta $900 \mathrm{~m}$ en la provincia de Azuay (Whittingham, 1994).

Esta especie prefiere espacios donde la vegetación es densa a orillas de arroyos, en parches de bosques tanto maduros como secundarios (Pople et al., 1997). Está categorizada como Vulnerable de extinción a nivel global y en Ecuador (Granizo et al., 2002; BirdLife International, 2016). Las poblaciones de O. occidentalis se encuentran en bajas densidades y muy fragmentadas, debido a presiones antropogénicas como la acelerada deforestación (Ridgely \& Greenfield, 2001; Granizo et al., 2002; Baquero et al., 2004; Flanagan et al., 2005). Solo se conoce aspectos generales de la biología reproductiva de la especie (e.g., cría y actividades de cotejo; Sample et al., 2016; del Hoyo et al., 2018).

Pezo, K. (2019). Nuevo registro de anidación y descripción del nido del Mosquero Real del Pacífico Onychorhynchus occidentalis (Passeriformes: Tyrannidae) en El Oro, Ecuador. Revista Ecuatoriana de Ornitología, 5, 9-13 
Es necesaria más información detallada y a escala local para mejorar el conocimiento sobre su historia natural. En este sentido, nuevos datos sobre la biología reproductiva, tiempos de nidificación y comportamiento reproductivo pueden ser relevantes para comprender sus requerimientos reproductivos. Aquí se presenta la primera nota de comportamiento reproductivo y descripción de nido de O. occidentalis al sur del Ecuador, en la provincia de El Oro.

El 7 de mayo de 2017 se observó el comportamiento de anidación de $O$. occidentalis en un bosquete rodeado de pastizales a $\sim 100 \mathrm{~m}$ de un riachuelo, durante un recorrido hacia la finca Tres Marías $(-3,687416,-79,909861$; 236 m s.n.m.; Fig. 1) en un bosque húmedo piemontano y semi-deciduo montano bajo (MAE, 2012). La hembra emitióvocalizaciones fuertes acompañadas de vuelos cortos, y a $2 \mathrm{~m}$ de distancia de ella se localizó un nido activo con un pichón. El nido estaba colgado de una fina rama de un árbol del género Erythrina a 4,1 m sobre el suelo. El área se caracterizaba por la presencia de árboles y arbustos densos de los géneros Phyllostylon, Triplaris, Guazuma, Leucaena, Inga y Trichanthera. El sotobosque estaba dominado por hierbas perennes y algunos helechos. El nido consistía de una estructura caediza-colgante, provista de una entrada en ovalo hacia el centro. Las dimensiones del nido fueron: i) diámetro interno $=7 \mathrm{~cm}$; ii) diámetro externo $=16 \mathrm{~cm}$; iii) alto $=$ $8 \mathrm{~cm}$; iv) profundidad $=6 \mathrm{~cm}$; v) diámetro de la entrada $=5 \mathrm{~cm}$. El peso seco del nido fue de 26,62 g.

Externamente el nido presentaba un tejido semi-abierto hecho básicamente de raíces secas de bromelias, hierbas entrelazadas, fibras de musgo seco y hojas secas en los extremos, con una cubierta donde destacaban ramas pequeñas (Fig. 2). En la parte interna, la estructura se mostraba mucho más compacta y con tejidos de material más fino. El piso del nido estaba cubierto con pedazos muy pequeños de hojas secas y algunas plumas pequeñas provenientes del pichón. Nidos con características similares fueron reportados por Whittingham (1994) en las estribaciones occidentales de la provincia del Azuay y por Berg (1994) en la provincia del Guayas. Del Hoyo et al., (2018) describen un nido incompleto, pero no mencionan la localidad del origen.

Al llegar la hembra adulta al nido con el alimento, tardó $\sim 5$ min en ingresar al nido. La aproximación al nido fue mediante vuelos cortos a manera de zigzag. Una vez en el nido, permaneció dentro por $\sim 5 \mathrm{~s}$, tiempo en el cual alimentó al pichón. Esta actividad de alimentación duró en total $\sim 40 \mathrm{~min}$ por día, con lapsos de $\sim 20 \mathrm{~min}$ entre salida y llegada al nido. La hembra se movió en un radio no mayor a $8 \mathrm{~m}$ del nido durante la vigilia. En la dieta del pichón se pudo diferenciar que la hembra llevó en su pico insectos de los órdenes Odonata (libélulas), Hemiptera (larvas de cigarra) y otros pequeños insectos no identificados, los cuales debían ser traídos de un radio no mayor a $\sim 1 \mathrm{~km}$ del nido. Esta distancia fue estimada debido a que la hembra fue vista en dos ocasiones durante recorridos hacia el sitio de nidificación. Este comportamiento se repitió durante los siguientes 5 días. A pesar de que el 10 de mayo apareció otro individuo, aparentemente la pareja reproductora, no se logró identificar el sexo ya que en ninguno de sus movimientos se pudo visualizar la coloración de la cresta. Durante el evento de alimentación se observó que mientras un individuo iba por alimento, el otro permanecía en vigilia. Para ello, ambos hacían vuelos cortos y vocalizaciones al momento de aproximarse al nido (i.e., kiiyu kiiyu), mientras el polluelo resonaba suavemente (i.e., ptrrr ptrrr). Desde el 12 de mayo, a las $16 \mathrm{~h} 15$, el nido se encontró vacío y en los posteriores 10 días no tuvo actividad alguna.

Este reporte es el primero para la provincia de El Oro y demuestra que $O$. occidentalis anida también en bosque secundario en primeros estados de sucesión. Esta observación lleva a considerar aquellas zonas en recuperación en estribaciones de la cordillera de los Andes como sitios convenientes para la reproducción de especies amenazadas. Sin embargo, estas zonas son susceptibles al cambio de uso de suelo debido a degradación y pérdida de la cobertura vegetal (Sierra, 2013), por lo que el monitoreo de nidos de especies amenazadas puede ser importante para mejorar el manejo y conservación de hábitat secundarios.

\section{AGRADECIMIENTOS}

Quiero expresar mi agradecimiento a la Universidad del Azuay por apoyar y fomentar la investigación. Agradecezco de manera especial la colaboración de Carlos Pezo Yaguana en la fase de campo y la recolección de datos; a Sebastián Padrón por el apoyo y por sus comentarios al manuscrito; a Carlos Nivelo y Boris Tinoco por facilitarme información; y a Pedro Astudillo Webster por sus valiosas sugerencias y ediciones durante el desarrollo de esta nota. 


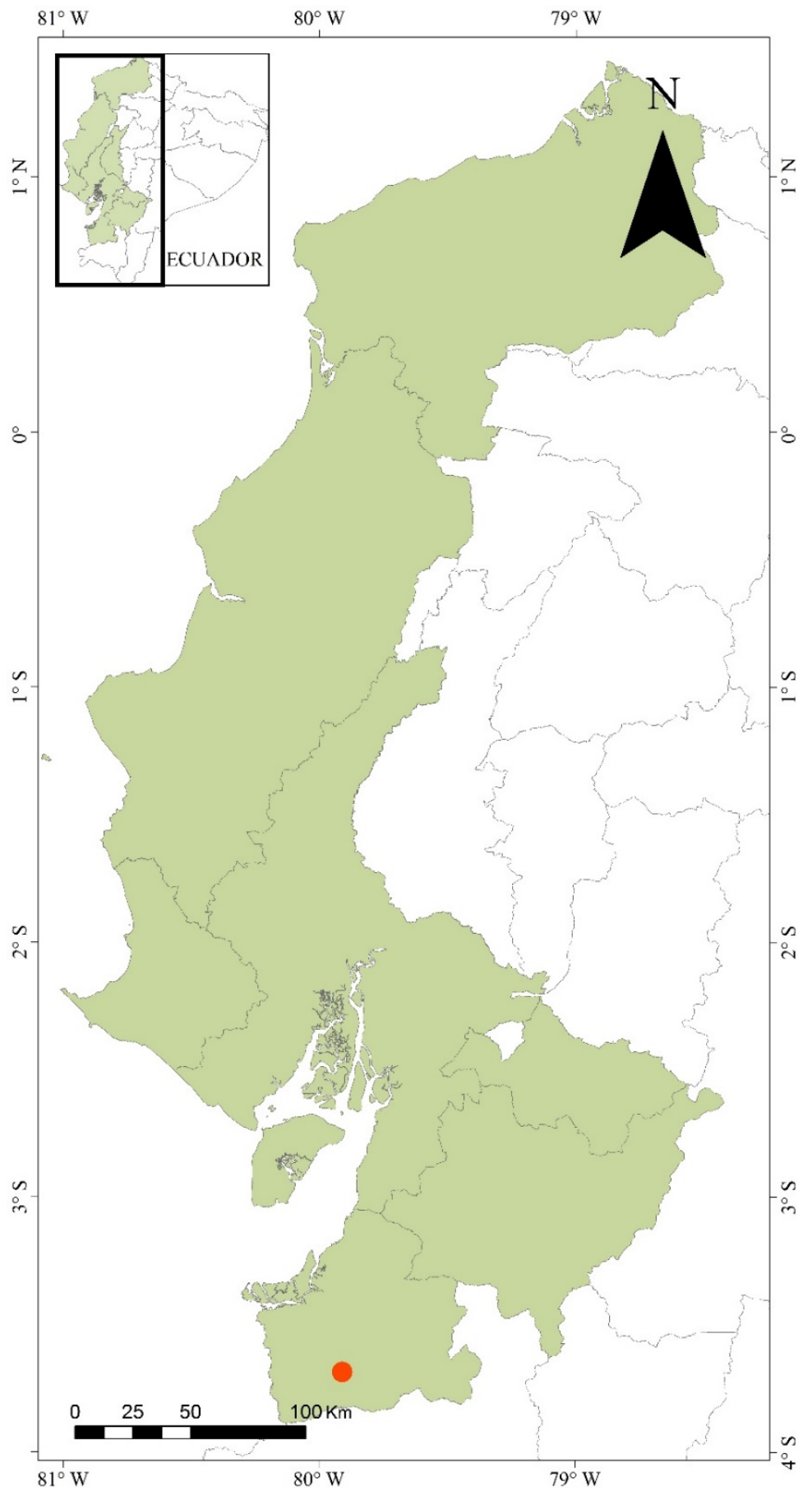

Figura 1. Distribución de Onychorhynchus occidentalis en el Ecuador. Área de color verde muestra la distribución aproximada en el país; el punto naranja corresponde al registro del evento reproductivo al sureste de El Oro. 

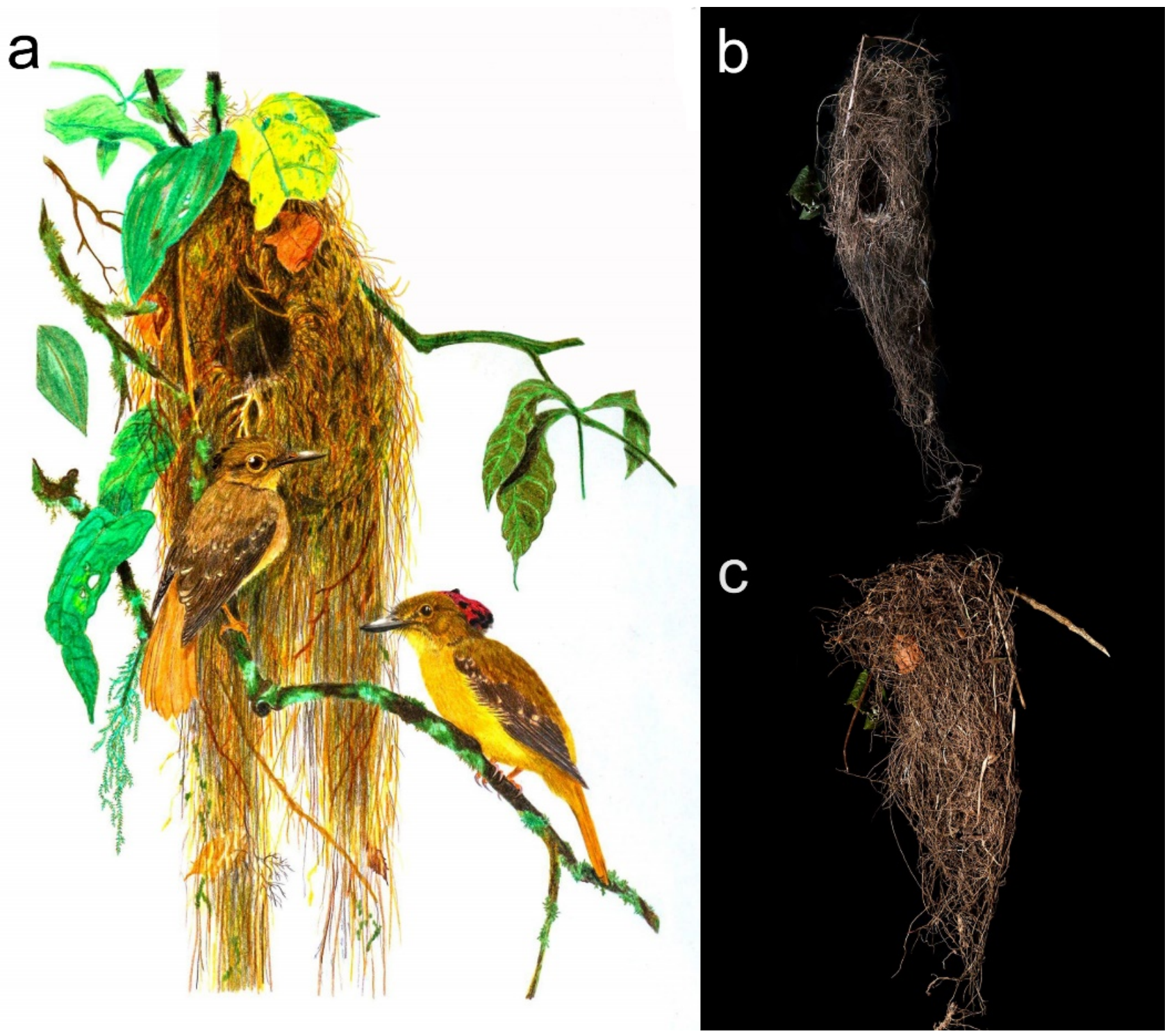

Figura 2. (a) Pareja de Onychorhynchus occidentalis en un nido activo en la provincia de El Oro, suroeste de Ecuador (José Falcón); (b-c) Vista frontal y lateral del nido encontrado.

\section{REFERENCIAS}

Baquero, F., Sierra, R., Ordóñez, L., Tipán, M., Espinoza, L., Rivera, M., \& Soria, P. (2004). La vegetación de los Andes del Ecuador: memoria explicativa de los mapas de vegetación potencial y remanente de los Andes del Ecuador a escala 1: 250.000 y del modelamiento predictivo con especies indicadoras. Quito, Ecuador: EcoCiencia.

Berg, K. (1994). New and interesting records of birds from a dry forest reserve in south-west Ecuador. Cotinga, 2, 14-19.

Best, B. J., Checker, M., Thewlis, R., Best, A., \& Duckworth, W. (1996). New bird breeding data from southwestern Ecuador. Ornitología Neotropical, 7, 69-73.

BirdLife International. (2016). Onychorhynchus occidentalis. The IUCN Red List of Threatened Species 2016: e.T22699653A93742094. http://dx.doi.org/10.2305/IUCN.UK.2016-

del Hoyo, J., Collar, N. \& Kirwan, G. (2018). Pacific Royal Flycatcher (Onychorhynchus occidentalis). In: J. del Hoyo, A. Elliott, J. Sargatal, D. A. Christie, \& E. de Juana (Eds.), Handbook of the birds of the world alive. Barcelona: Lynx Edicions. https://www.hbw.com/node/1343671. 
Flanagan, J., Franke, I., \& Salinas, L. (2005). Aves y endemismo en los bosques relictos de la vertiente occidental andina del norte del Perú y sur del Ecuador. Revista Peruana de Biología, 12(2), 239-248.

Granizo, T., Pacheco, C., Ribadeneira, M., Guerrero, M., Suárez, L. (Eds.). (2002). Libro rojo de las aves del Ecuador. Quito, Ecuador: SIMBIOE, Conservación Internacional, EcoCiencia, Ministerio del Ambiente \& UICN, Serie Libros Rojos del Ecuador, tomo 2.

Ministerio del Ambiente del Ecuador. (2012). Sistema de clasificación de los ecosistemas del Ecuador continental. Quito, Ecuador: Subsecretaría de Patrimonio Natural.

McMullan, M., \& Navarrete. L. (2017). Fieldbook of the birds of Ecuador. Quito, Ecuador: Ratty Ediciones.

Pople, R. P., Burfield, I., Clay, R. P., Cope, D., Kennedy, C., López-Lanús, B., \& Warren, B. (1997). Project Ortalis' 96: interesting and important results of bird surveys in western Ecuador. Cotinga, 8, 59-63.

Ridgely, R. S., \& Greenfield, P. J. (2001). The birds of Ecuador. Vol. 1: status, distribution and taxonomy. Ithaca, New York: Cornell University Press.

Sample, R., Shackelford, B., \& Kannan. R. (2016). Royal Flycatcher (Onychorhynchus coronatus). In: T. S. Schulenberg (Ed.), Neotropical Birds Online. Ithaca, New York: Cornell Lab of Ornithology. https://neotropical.birds.cornell.edu/Species-Account/nb/species/royfly1

Sierra, R. (2013). Patrones y factores de deforestación en el Ecuador continental, 1990-2010 y un acercamiento a los próximos 10 años. Quito, Ecuador: Conservación Internacional Ecuador y Forest Trends.

Vázquez, M. A., Larrea, M., Suárez, L., \& Ojeda, P. (Eds.). (2001). Biodiversidad en Los bosques secos del suroccidente de la provincia de Loja: un reporte de las evaluaciones ecológicas y socioeconómicas rápidas. Quito, Ecuador: EcoCiencia, Ministerio del Ambiente, Herbario LOJA \& Proyecto Bosque Seco.

Whittingham, M. (1994). Observations at a nest of Pacific Royal Flycatcher Onychorhynchus coronatus occidentalis. Bulletin of the British Ornithologists' Club, 114, 131-132. 